\title{
New 3D technology to help in the battle against brain tumours
}

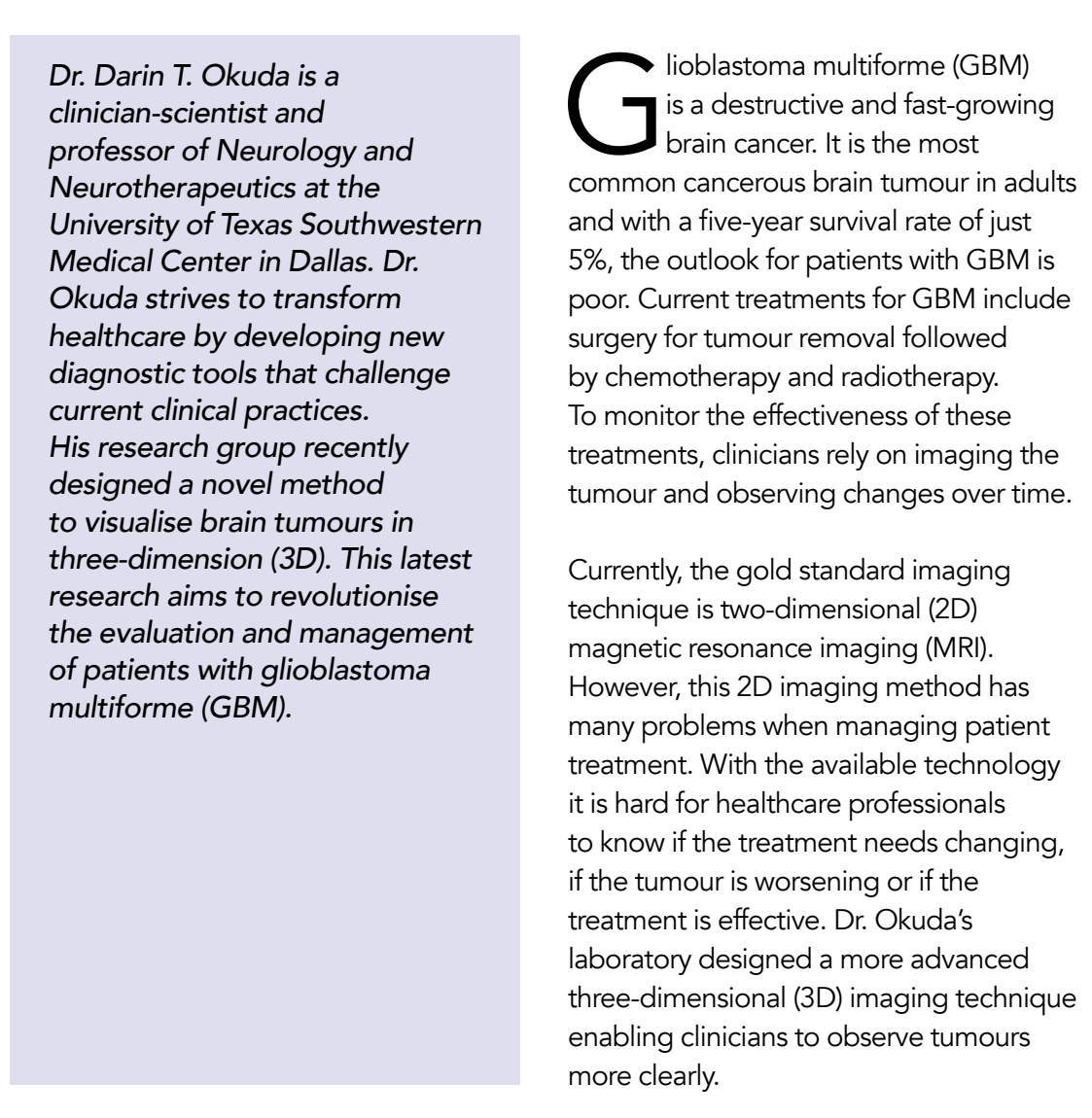

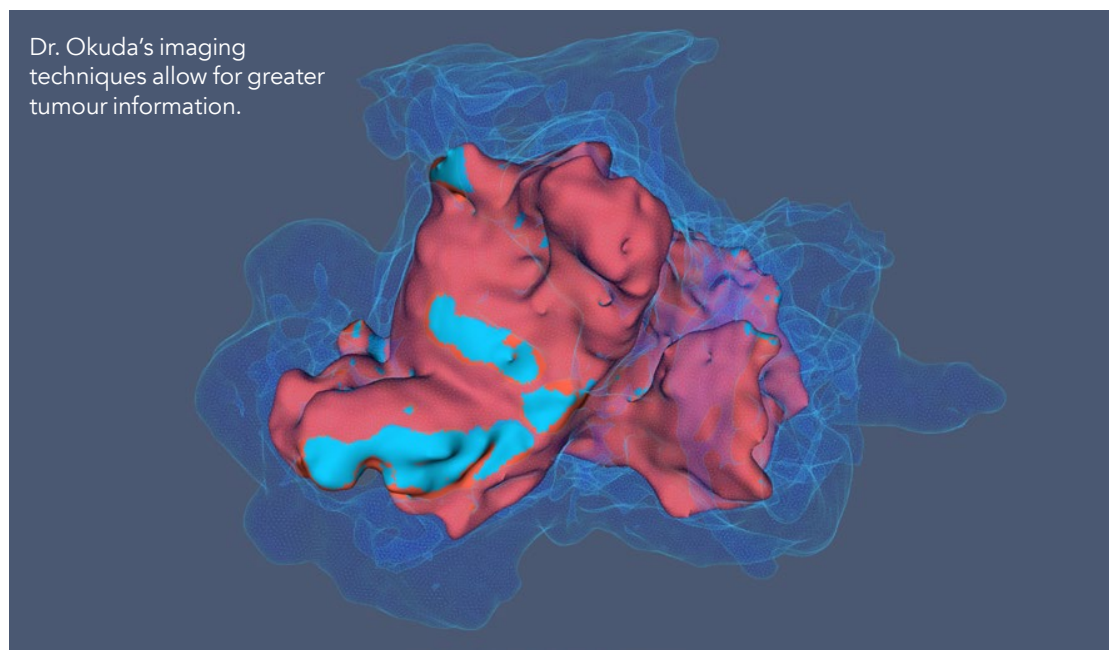

\section{HOLDING US BACK}

Studying the brain is notoriously difficult as access to the brain is very trickyespecially when trying to study the brains techniques have bifferent imaging scientists peek inside the brain o help these is $2 \mathrm{D} \mathrm{MRI}$. This technique is useful for imaging tumours like GBM. These 2D MR images are currently used to help healthcare providers monitor the effects of treatments on tumours.

During MRI, a chemical called gadolinium is injected into the body to enhance and improve the visualisation and understanding of how leaky blood vessels are. Clinicians use these images to monitor GBM development. Currently it is accepted amongst healthcare professionals that an increase in the Min contrast signal, known as a contrast our growth.

This sounds simple - when the contrast enhancement increases, the tumour not that easy to interpret what is going on in a 2D MRl: there are important limitations to take into account Treatm for GBM, such as radiotherapy, can cause he contrast enhancement on the MRI. This phenomenon is known as pseudoprogression and it is often indistinguishable from tumour growth. This makes it very difficult for clinicians to understand the true nature of the fumour and provide the best treatment for their patients.

In order to treat GBM patients effectively, It is vital that tumour growth be viewed accurately. These $2 \mathrm{D}$ forced perspective
MRls limit the understanding of tumour

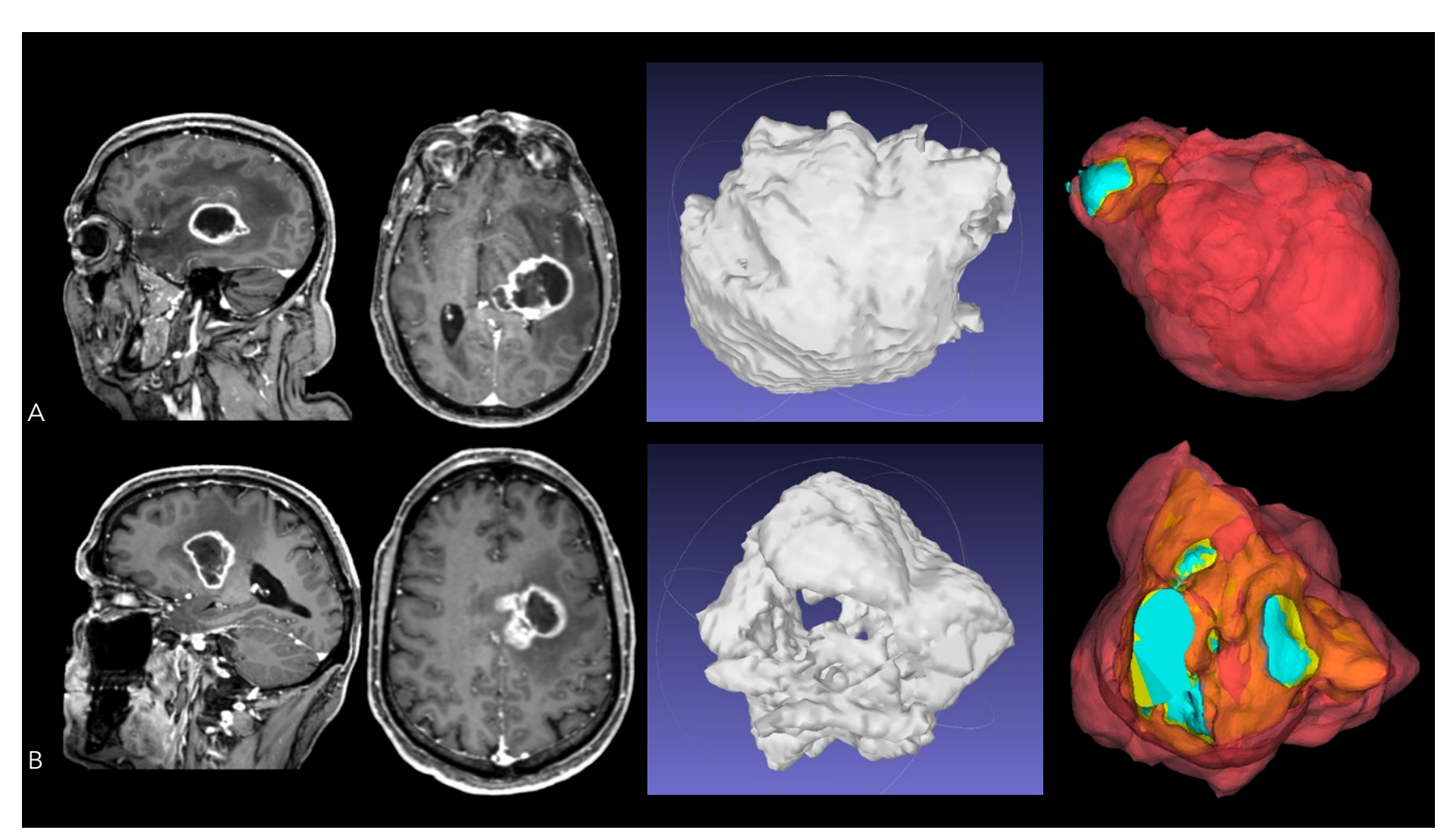

The contrast is clear between $2 \mathrm{D} \mathrm{MRI}$ (left), the initial $3 \mathrm{D}$ image (centre) and data from the coverage analysis (far right). The patient in the top row exhibits

growth and fail to reveal the complexity of the contrast enhanced region to reveal details about the tumours in a way that
had never been seen before. The

thickness of the contrast enhanced of the tumour, specifically the shape and texture. Dr. Okuda's laboratory help uncover the true characteristics of GBM tumours.

SEEING THINGS DIFFERENTLY Dr. Okuda's research group was able to reveal 3D spatial, structural and surface features of GBM using a novel approach. outer shell was different between

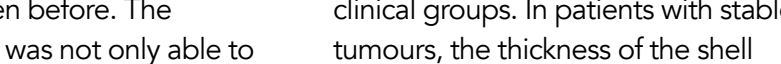
effectively isolate the contrast enhanced was found to be more evenly spread portion of the tumour, but it was able to edit the acquired images in 3D, an aspect not previously avaliable with oth plattorms. Three different approaches compared to worsening tumours. The shells also tended to be thinner than in those patients with tumour growth. Scientists are still trying to understand whether the width of the shell changes during tumour growth - more research In a recent study fitteen patients divided into two groups: 1) clinically stable without new or worsening neurological symptoms and

Dr Okuda's laboratory have designed wuld be needed a more advanced 3D imaging technique group suggests that enabling clinicians to observe tumours patients overtime

2) patients with new or worsening neurological symptoms unattenuated by steroid treatment whose combined symptoms and MRI changes warranted a change in their established treatment plan. Each patient had MRI scans and the resulting $3 \mathrm{D}$ images were studied and the two groups compared. The researchers started by investigating the most active region of the tumour, the

The software that Dr. Okuda's group developed allowed in-depth analysis

\begin{abstract}
more clearly.
\end{abstract}
A technique known as 'principle component analysis' was used to understand more about the shape The outer shell of the and the dictive region was analysed axes was studied to reveal the tumour's sphericity. The researchers predicted which were getting worse would be more spherical in shape compared to stable tumours.

The researchers used 'medial axis transformation' to determine if the patients over tim
may be more

meaningful when looking at outer shell width during disease progression. This new technology could help healthcare their patient's tumour changes over time.

'Coverage analysis' was used to identify the number of holes in the tumour's relationship between the inner and outer The restand he integnty of this region. The researchers found hat in patients with worsening tumours there were fewer contrast enhanced outer shell. The surfaces of the tumour were studied to 


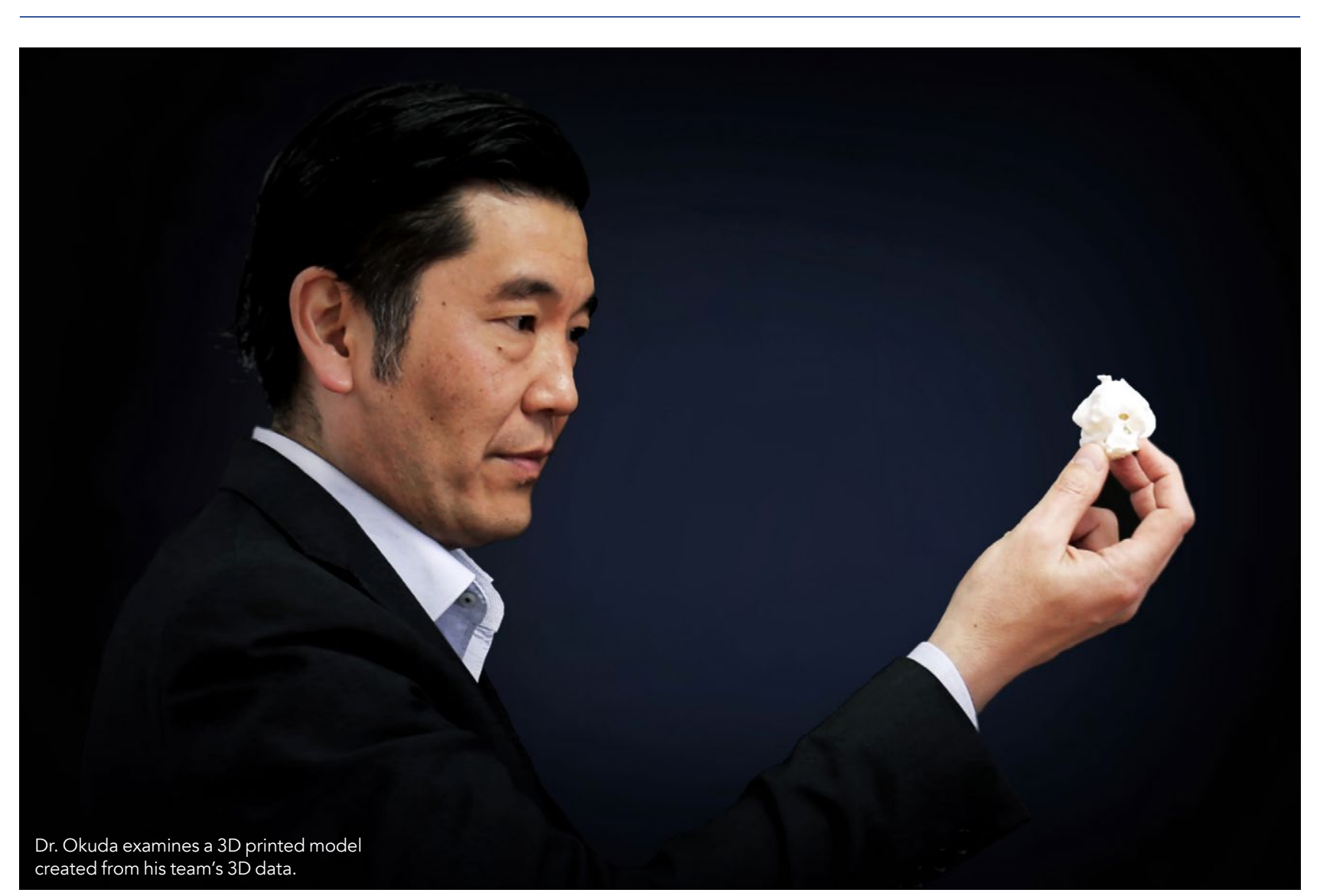

with stable tumours. The research group had expected to see this in a growing tumour because with nothing to prevent tumour growth, the cells migrate outwards resulting in a more spherical, fuller shape with less holes.

In combination, these three analysis techniques bring together a $3 \mathrm{D}$ picture of a tumour's shape and texture. The 3D data was able to provide much more thorough information than the the researchers were able to show that the changes in 3D spatial structurat and suface features correlated with the clinical status of the tumour. This $3 D$ morphological data provides a vital tool monitoring tumour progression in patient in order to provide better treatment.

\section{WHAT NEXT}

This emerging 3D technology provides new hope for GBM patients. The

$3 \mathrm{D}$ view of the tumour extends way beyond the traditional, constrained 2D images and provides more dynamic and comprehensive information about the tumour. This 3D technology will allow healthcare providers to gain new gain a gro tur rour prograssion nawd

This 3D morphological data provides a vital tool in monitoring tumour progression in patients in order to provide better treatment.

advancement based on shape, texture simulations of the tumour could be in trament of patients and any chang . could then see the exact size and shape of the tumour. this is much more tangible acquired 3D files may a augmented and virtual reality plafforms.

For clinicians and patients, being able to and true tumour progression is key. The technology could also be used to monitor such as immune-based therapeutic strategies which harness the immune immunotherapy is well established for some cancers, it is still being evaluated for the treatment of GBM. It is possible that this $3 \mathrm{D}$ technology could play a part in evaluating the therapeutic effect of such treatments.

Dr. Okuda's research group is currently conducting studies using larger patient groups to confirm their findings and ultimately aim to measure the impact of their findings on patient care. The group also hopes to use 3D data to identify disease progression before symptoms manifest in patients by leveraging machine and deep learning techniques. These efforts extend beyond the curent routhe disease survellance measures and their methodology Not only is this $3 \mathrm{D}$ technology useful may one day be systematically applied in monitong tumours and treatment of to the managementof

for patient education. These $3 \mathrm{D}$ computer and structural patterns. This will aid better printed to create a 3D model. The patien the effectiveness of various treatments system to fight cancerous tumours. While

\section{Behind the Research} Dr. Darin T. Okuda

: Darin.Okuda@UTSouthwestern.edu T: +12146458215 (office) W: https://profiles.utsouthwestern.edu/profile/146752/darin-t-okuda.htm

\section{Research Objectives}

Dr. Darin T. Okuda and his team from UT Southwestern Medical Center in Dallas developed novel methodology to visualise brain tumours in $3 \mathrm{D}$.

\section{Detail}

Darin T. Okuda, M.D., M.S., F.A.A.N., F.A.N.A. Professor of Neurology \& Neurotherapeutics

Sclerosis \& Neuroimmunology Imaging

Director, Radiologically Isolated Syndrome Consortium Department of Neurology \& Neurotherapeutics UT Southwestern Medical Center at Dallas 5959 Harry Hines Blvd. USA

Dr. Okuda is a clinician-scientist and professor of neurolog specialising in neuroimmunology. His work aims to create

next generation heathcare by developing innovalive connections between patients and providers that transform medical management. He is involved in the implementation of extreme healthcare concepts focused on delivering car

Collaborators

- Edward Pan

- Andrew Wilson

- Morgan McCreary

- YeqiWang

- Thomas Stanley

- Marco C. Pinho

- Xiaohu Guo

UTSouthwestern Medical Center

\section{References}

Hansen M, Pan E, Wilson A, McCreary M, Wang Y, Stanley T, Pinho M, Guo X, Okuda D. (2018). Post-gadolinium 3-dimensional spatial, surface, and structural characteristics of glioblastomas differentiate pseudoprogression from true tumor progression. Journal of Neuro-Oncology, 139(3), 73

Newton B, Wright K, Winkler M, Bovis F, Takahashi M, Dimitrov I, Sormani M, Pinho M, Okuda D. (2017). ThreeDimensional Shape and Surface Features Distinguish Multiple Journal of Neuroimaging, 27(6), 613-619.

Okuda D. (2018). 2018 American Academy of Neurology watch?v=w_4aAVfyzq8\&feature $=$ youtu. be

\section{Personal Response}

What difference will this make to brain tumo treatment from a patient perspective?

II Our work allows for patients to be better educated on their condition as the acquired $3 \mathrm{D}$ images and models from tumours are immediately intuitive. This approach surpasses education ha is pros These tumour advancement as compared to treatment effects, the prevention of unnecessary neurosurgical procedures from being performed, and for treatment changes to occur more quickly. In addition, our 3 D approach provides a more of new and novel GBM treatments for our patients. in larger groups, will allow for the earlier recognition of 